\title{
REVIEW ARTICLE OPEN \\ Reduced focal fiber collinearity in the cingulum bundle in adults with obsessive-compulsive disorder
}

\author{
A. Versace (D) ${ }^{1}$, S. Graur ${ }^{1}$, T. Greenberg ${ }^{1}$, J. P. Lima Santos ${ }^{1}$, H. W. Chase ${ }^{1}$, L. Bonar ${ }^{1}$, R. S. Stiffler ${ }^{1}$, R. Hudak ${ }^{1}$, Tae Kim² ${ }^{2}$ A. Yendiki ${ }^{3}$, \\ B. Greenberg ${ }^{4}$, S. Rasmussen ${ }^{4}$, H. Liu (D) ${ }^{3}$, S. Haber ${ }^{5}$ and M. L. Phillips ${ }^{1}$
}

\begin{abstract}
Obsessive-compulsive disorder (OCD) is a disabling condition, often associated with a chronic course. Given its role in attentional control, decision-making, and emotional regulation, the anterior cingulate cortex is considered to have a key role in the pathophysiology of the disorder. Notably, the cingulum bundle, being the major white matter tract connecting to this region, has been historically a target for the surgical treatment of intractable OCD. In this study, we aimed to identify the extent to which focal - more than diffuse-abnormalities in fiber collinearity of the cingulum bundle could distinguish 48 adults with OCD (mean age $[S D]=23.3$ [4.5] years; $F / M=30 / 18$ ) from 45 age- and sex-matched healthy control adults (CONT; mean age [SD] = 23.2 [3.8] years; $\mathrm{F} / \mathrm{M}=28 / 17$ ) and further examine if these abnormalities correlated with symptom severity. Use of tract-profiles rather than a conventional diffusion imaging approach allowed us to characterize white matter microstructural properties along (100 segments), as opposed to averaging these measures across, the entire tract. To account for these 100 different segments of the cingulum bundle, a repeated measures analysis of variance revealed a main effect of group $\left(\mathrm{OCD}<\mathrm{CONT} ; \mathrm{F}_{[1,87]}=5.3 ; P=0.024\right)$ upon fractional anisotropy (FA, a measure of fiber collinearity and/or white matter integrity), in the cingulum bundle, bilaterally. Further analyses revealed that these abnormalities were focal (middle portion) within the left and right cingulum bundle, although did not correlate with symptom severity in OCD. Findings indicate that focal abnormalities in connectivity between the anterior cingulate cortex and other prefrontal cortical regions may represent neural mechanisms of OCD.
\end{abstract}

Neuropsychopharmacology (2019) 44:1182-1188; https://doi.org/10.1038/s41386-019-0353-4

\section{INTRODUCTION}

Obsessive-compulsive disorder (OCD), characterized by intrusive thoughts and time-consuming compulsive behaviors [1], is often associated with a chronic and severe course that can lead to severe social and functional impairments [1]. The prevalence of OCD was previously underestimated [1]; more recent estimates indicate a lifetime prevalence of $2.3 \%$ in the general population [1], and a high comorbidity with other psychiatric disorders, including anxiety, depression, impulse-control, and substance use disorders [2]. While selective serotonin reuptake inhibitors (SSRIs) and behavioral therapy are effective treatments for the disorder, $\sim 30 \%$ of patients with OCD do not respond adequately to conventional therapies [3].

As a result of the failure of a sizeable proportion of the OCD population to respond to conventional treatments, focal lesions (for review see ref. [4]), and, more recently, high-frequency stimulation of neural regions implicated in the pathophysiology of OCD have been advocated as viable alternatives for treatmentresistant patients with $O C D$ [5]. One neural region that has historically been a target for focal lesions as a treatment for OCD is the anterior cingulate cortex (ACC), given its role in attentional control, decision-making, and emotional regulation [6], processes that are abnormal in OCD [7].
A prospective analysis of 198 patients with bilateral lesions of the ACC-bilateral cingulotomy-showed lasting improvements in approximately half of those treated for OCD [8], where improvements persisted for at least 2 years [8]. The relief that has been reported by OCD (and other psychiatric) patients after cingulotomy is striking [9]. Interestingly, clinical improvement does not appear to originate from a direct effect of the cingulotomy procedure on reducing OCD symptom severity, but rather result from a reduction of attention to negative emotional thoughts [10]. Indeed, one study reported that negative emotional and obsessional thoughts still occurred in individuals with OCD after cingulotomy, but that such thoughts were no longer bothersome [11]. A clearer understanding of the neural mechanisms underlying OCD symptomatology is needed to elucidate the more focal neural abnormalities that reflect pathophysiologic mechanisms of OCD, and that can act as targets to guide and improve intervention strategies for the treatment of this debilitating disorder.

One way forward to increase understanding of the neural mechanisms underlying $O C D$ is to focus more on the neural circuitry of OCD by examining the key white matter tracts that connect the ACC with other neural regions shown to be functionally abnormal in OCD. These regions include prefrontal cortical regions, such as those implicated in attentional control

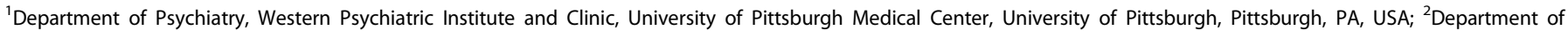

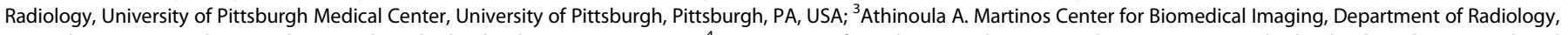

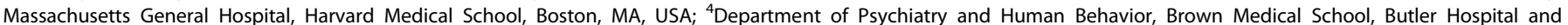
Providence VA Medical Center, Providence, RI, USA and ${ }^{5}$ Department of Pharmacology and Physiology, University of Rochester Medical Center, Rochester, NY 14642 , USA Correspondence: A. Versace (versacea@upmc.edu)

Received: 30 November 2018 Revised: 17 January 2019 Accepted: 4 February 2019

Published online: 25 February 2019 
and set shifting (e.g., dorsolateral and ventrolateral prefrontal cortex) [12] or in value encoding (i.e., orbitofrontal cortex) [13], as well as subcortical regions primarily involved in mediating the function of the ACC and prefrontal cortical regions via frontostriatal-thalamic-frontal circuits [14]. The combination of functional abnormalities in the ACC and these regions is thought to be associated with the difficulty in disengaging from otherwise nonsalient stimuli or contexts, i.e., deficits in set shifting, and the development of obsessional thoughts and compulsive behaviors that characterize the disorder [7].

A white matter tract that carries fibers from all of these regions is the cingulum bundle, one of the most distinctive long associative tracts $[6,15,16]$. Indeed, a large number of diffusion imaging studies has consistently reported white matter structural abnormalities in the cingulum bundle in individuals with OCD (for a recent review of 17 OCD studies see ref. [17]; for a recent metaanalysis of 22 OCD studies see ref. [18]). The majority of these studies reported lower fractional anisotropy (FA), or greater mean/ radial diffusivity, reflecting lower fiber collinearity in this tract in adults with OCD relative to healthy adults. Other studies, mostly in children [19-21], reported abnormal increases in FA in this tract, indicating that important developmental abnormalities may occur in the cingulum bundle in individuals with OCD. Indeed, an age effect was reported in a more anterior portion of the cingulum, where greater reductions in FA were reported in older than younger adults [22-24]. Greater mean diffusivity (i.e., lower collinearity or FA) in the left cingulum bundle was also associated with greater severity of OCD symptoms in adults with OCD [25], and greater FA in the left cingulum bundle with better performance on directed attention (Stroop Test) and executive control (Trails Making Test) tasks [19] in children with OCD, suggest that microstructural abnormalities in this tract might underlie development of some of the symptoms of OCD.

Abnormalities in other white matter tracts have also been reported in OCD. Abnormal (decreased or increased) FA has been reported in the genu $[20,26,27]$, body $[28,29]$, and splenium $[27$, 30] of the corpus callosum and/or anterior projecting fibers, including the anterior corona radiata, anterior thalamic radiation, and/or anterior limb of the internal capsule [21, 25, 31, 32]. These findings are less consistent across studies than those regarding the cingulum bundle, however. This inconsistency can in part be explained by the heterogeneity of the disorder, and methodological differences existing across studies. In this regard, it is important to note that the majority of these studies acquired relatively low-resolution diffusion imaging data, mostly fewer than 30 gradients in 1.5 Tesla scanners, and used voxel-based approaches, including voxel-based morphometric analyses or tract-based spatial statistics (TBSS). While valuable for the study of white matter microstructure across the whole brain, these approaches are of limited utility for the study of the integrity of specific white matter tracts, where tractography studies are better suited. Furthermore, the structure of the cingulum bundle is complex, comprising both short (U-fibers) and long sagittal associative fibers that link medial parts of the frontal, parietal, and temporal lobes $[6,33]$, and projection fibers that radiate across the tract to cortical and subcortical regions [33]. As such, averaging diffusivity measures across the entire cingulum bundle, as performed in conventional tractography studies, might not capture the complex architecture of this tract.

The goal of the present study was thus to employ, for the first time, a tract-profile approach in the study of the white matter microstructure of the cingulum bundle in adults with $O C D$ in comparison with healthy adults. Here, we used the Automated Fiber Quantification (AFQ; https://github.com/yeatmanlab/AFQ) toolbox to automatically reconstruct the cingulum bundle in each participant in native space. AFQ allows the derivation of "tractprofiles" that measure diffusivity properties at sample positions (e.g., 100 segments) from the start to the end of each reconstructed tract. Use of tract-profiles-rather than conventional approaches measuring white matter microstructure across an entire tract-allowed us to determine the extent to which white matter microstructural abnormalities previously reported in OCD were localized to focal portions (i.e., 5 or more consecutive segments) of the cingulum bundle rather than being widespread along the entire tract. This approach also allowed us to further examine the extent to which any focal abnormalities were associated with specific OCD symptomatology. We hypothesized that adults with OCD would show significantly reduced FA in focal regions of the cingulum relative to healthy adults, and specifically in portions of the tract carrying fibers from neural regions previously shown to be functionally abnormal in OCD, including the ACC, orbitofrontal cortex, dorsolateral prefrontal cortex, and subcortical nuclei. We further hypothesized that these abnormalities would be significantly associated with OCD symptom severity, where greater FA deceases would be associated with greater symptom severity. Additional major white matter tracts were also examined in exploratory analyses.

\section{METHODS}

Participants

Ninety-three adults including 48 adults with $O C D$ (age range $=$ 18-35 years mean age $[S D]=23.3$ [4.5] years; $F / M=30 / 18$ ) and 45 age- and sex-matched healthy adults (age range $=18-33$ years; mean age $[S D]=23.2$ [3.8] years; $F / M=28 / 17$ ) were included in the study. There were no between-group differences in socioeconomic status (SES) and predicted IQ, as measured using the National Adult Reading Test-revised [34] (Table 1). Six out of 48 adults with OCD had a comorbid diagnosis of major depressive disorder (MDD) at the time of the scan. Recruitment period was 6/3/2015-10/20/2018.

Inclusion criteria in all participants were 18-35 years of age and right-handedness. All participants were interviewed using the Structured Clinical Interview for DSM-5. Adults with OCD diagnosis without predominant hoarding symptoms and Yale-Brown Obsessive-Compulsive Scale (Y-BOCS) [35] score $>16$ were included in the study. Exclusion criteria in all participants were personal/family history (1st/2nd degree relatives) of schizophrenia/schizoaffective disorder, other primary psychotic disorders, bipolar disorder; present posttraumatic stress disorder; present suicidal ideation; present psychotic symptoms; personal history of head injury, neurological, neurodevelopmental (e.g., autism), tic disorders, systemic medical (metabolic, endocrine, chronic inflammatory, vascular, autoimmune) disease from medical records and selfreport, all of which may confound interpretation of neuroimaging measures; MMSE score $<24$; premorbid IQ estimate $<85$; visual disturbance $(<20 / 40$ corrected Snellen visual acuity); left/mixed handedness; current, and history in the last 3 months of, alcohol and illicit substance abuse/dependence, determined by urine screen and clinical assessment of alcohol and substance use; current suicidal ideation, as assessed using the Hamilton Rating Scale for Depression, contraindications to MRI: metallic foreign objects, e.g., aneurysm clips/ pacemakers, or questionable history of metallic fragments, prone to panicking in enclosed spaces; positive pregnancy test for females/self-reporting of pregnancy. In adults with OCD, additional exclusion criteria were Y-BOCS score $<16$; predominant hoarding symptoms, given that hoarding may have a distinct neural circuitry [36]. In healthy adults, additional exclusion criteria were personal history of Axis I disorder, or substance abuse/dependence, family history (1st/2nd degree relatives) of OCD, neurodevelopmental disorder, schizophrenia and/or schizoaffective disorder, primary psychotic disorder, bipolar disorder, and present PTSD.

\section{Symptom assessment}

Type and severity of depressive, anxiety and obsessive-compulsive symptoms were measured using clinical rating scales and self- 
Table 1. Between-group differences in demographic and clinical variables

\begin{tabular}{|c|c|c|c|c|c|c|c|c|c|c|c|c|}
\hline Variables & Group & $N$ & & & & & & Mean & SD & $t / x^{2}$ & df & $P($ two-tailed $)$ \\
\hline & OCD & 6 & 42 & & & & & - & - & & & \\
\hline & OCD & 48 & & & & & & 23.3 & 4.5 & & & \\
\hline $\operatorname{Sex}(F / M)$ & CONT & 28 & 17 & & & & & - & - & 0.1 & 1 & 0.978 \\
\hline & OCD & 1 & 9 & 20 & 0 & 14 & 4 & - & - & & & \\
\hline \multirow[t]{2}{*}{ Predicted full IQ } & CONT & 45 & & & & & & 109.9 & 0.9 & & 91 & 0.387 \\
\hline & OCD & 48 & & & & & & 111.1 & 5.8 & & & \\
\hline \multirow[t]{2}{*}{ HRSD17 } & CONT & 45 & & & & & & 1.2 & 1.2 & 11.6 & $51^{a}$ & $<0.001$ \\
\hline & OCD & 48 & & & & & & 10.8 & 5.6 & & & \\
\hline \multirow[t]{2}{*}{ OC-TCDQ harm avoidance } & CONT & 45 & & & & & & 3.1 & 4.9 & 14.7 & $80^{\mathrm{a}}$ & $<0.001$ \\
\hline & OCD & 48 & & & & & & 23.0 & 7.9 & 14.7 & 80 & \\
\hline \multirow[t]{2}{*}{ OC-TCDQ incompleteness } & CONT & 45 & & & & & & 4.4 & 4.9 & 11.4 & $70^{\mathrm{a}}$ & $<0.001$ \\
\hline & OCD & 48 & & & & & & 22.7 & 9.9 & 11.4 & 70 & \\
\hline \multirow[t]{2}{*}{ POPS } & CONT & 36 & & & & & & 102.5 & 33.6 & 9.3 & 72 & $<0.001$ \\
\hline & OCD & 38 & & & & & & 180.6 & 38.4 & & & \\
\hline \multirow[t]{2}{*}{ Difficulty with change } & CONT & 36 & & & & & & 18.2 & 7.3 & 8.4 & 72 & $<0.001$ \\
\hline & OCD & 38 & & & & & & 33.9 & 8.7 & & & \\
\hline \multirow[t]{2}{*}{ Emotional overcontrol } & CONT & 36 & & & & & & 16.1 & 5.9 & 4.9 & 64 & $<0.001$ \\
\hline & OCD & 38 & & & & & & 24.8 & 9.1 & & & \\
\hline & OCD & 38 & & & & & & 3.1 & 3.0 & & & \\
\hline \multirow{2}{*}{$\begin{array}{l}\text { Temperament and Character Scale- } \\
\text { Harm Avoidance: Fear of uncertainty } 2\end{array}$} & CONT & 36 & & & & & & 4.1 & 1.5 & 5.3 & $72^{\mathrm{a}}$ & $<0.001$ \\
\hline & OCD & 38 & & & & & & 1.8 & 2.1 & 0.9 & & \\
\hline \multirow[t]{2}{*}{ Taking any medications [yes/no] } & CONT & $0 / 43$ & & & & & & 15.0 & & & 1 & $<0.001$ \\
\hline & OCD & $18 / 30$ & & & & & & & & & & \\
\hline
\end{tabular}

report questionnaires (for more details, see Supplemental Material)

Eighteen out of 48 adults with OCD were taking medications at the time of the study. These included antidepressant medications (SSRIs). The study was approved by the Institutional Review Board of the University of Pittsburgh. After being informed on the nature and aims of the study, all participants signed the consent form before participating in the study procedures.
Data analysis

Neuroimaging. Images were acquired on a 3T Siemens Magnetom Prisma (42 OCD and 40 CONT) and a 3T Siemens Magnetom Trio (6 OCD and 5 CONT) at the Magnetic Resonance Research Center, University of Pittsburgh Medical Center Health System, USA. Parameters of the dMRI acquisition are described in the Supplemental Material. Diffusion-weighted images were corrected for eddy current, subject motion and EPI distortion using topup and eddy 
Table 2. Two factorial repeated measure ANCOVA in the cingulum bundle in 48 adults with OCD and 45 healthy adults

\begin{tabular}{llc}
\hline Tests of between-subjects effects & $\mathrm{F}_{[1,87]}$ & Sig. \\
Group & 5.3 & 0.024 \\
Scanner & 16.1 & $<0.001$ \\
Age $[\mathrm{YY}]$ & 8.1 & 0.006 \\
Sex [M/F] & 1.1 & 0.295 \\
Tests of within-subjects effects & $\mathrm{F}_{[1,87]}$ & Sig. \\
Laterality & 3.9 & 0.050 \\
Laterality ${ }^{*}$ group & 0.1 & 0.767 \\
Tests of within-subjects effects & $\mathrm{F}_{[5,426]}$ & Sig. \\
Segment & 5.4 & $<0.001$ \\
Segment * group & 1.6 & 0.154 \\
Tests of within-subjects effects & $\mathrm{F}_{[7,596]}$ & Sig. \\
Laterality * group * segment & 1.1 & 0.364 \\
\hline Age and gender were covariates in all analyses & \\
Two factorial analysis included: Laterality (2 levels: left and right) and \\
segment (100 levels) & & \\
\hline
\end{tabular}

$[37,38]$, within FMRIB's Software Library (FSL). Six movement parameters, including average volume-by-volume translation and rotation in the $x, y$, and $z$ plane, were computed as previously proposed [39] in each participant. Between-group t-tests did not reveal any significant difference (Supplemental Table 1).

Data analysis and reconstruction of the cingulum tractogram were performed using the freely available Automated Fiber Quantification (AFQ) package (https://github.com/yeatmanlab/ $\mathrm{AFQ} /$ wiki). The tensor model was fitted to the $b=1000$ data to compute FA. AFQ uses a multi-step reconstructing procedure, including whole brain tractography, tract segmentation based on well-validated tracking protocols, tract refinement based on a probabilistic fiber tract atlas (Supplemental material for more details), and tract-profile calculation. The FA values are averaged in cross-sections (i.e., segments) along the left and right cingulum bundle in each participant. For a detailed characterization of FA along the tract, we used 100 segments [40]. Other tensor-based measures, namely axial and radial diffusivity ( $A D, R D)$, represent the displacement of water along the principal and non-principal diffusion directions, and can help interpret FA abnormalities [41]. Thus, $A D$ and $R D$ were also extracted along the left and right cingulum bundle in each participant.

In this tract of interest approach, left and right corticospinal tracts were examined as control tracts. While the focus of this study was on the cingulum bundle, additional major white matter tracts, including forceps minor/forceps major of the corpus callosum, anterior thalamic radiation, inferior longitudinal fasciculus, parietal and temporal portion of the superior longitudinal fasciculus and uncinate fasciculus were examined in exploratory analyses.

Statistical approach. Demographic, clinical, and diffusion imaging measures were imported into SPSS(v24) to test main hypotheses and exploratory analyses. Given the known effect of age (linear effect) and sex on white matter, these variables were covariates in all analyses. Scanner was an additional covariate in analyses.

To test our hypotheses, we adopted the following analytic approach.

Level-1 analysis: Using a two-way factorial repeated measure analyses of covariance (ANCOVA), we examined the main effect of group (OCD and CONT) on FA of the left and right cingulum bundle. Specifically, LATERALITY with 2 levels (1. LEFT; 2. RIGHT) and SEGMENTS with 100 levels were the within subject factors.
Level-2 analysis: Post-hoc ANCOVAs were employed in adults with OCD versus CONT on FA in each segment showing a significant effect of GROUP or GROUP-related interaction in Level1 analyses. To account for multiple comparisons of all $200 \mathrm{seg}$ ments, i.e., the $100 \times 2$ segments extracted from the tract-profiles of the left and right cingulum bundles, a method for multiple hypothesis testing for possibly dependent tests was used. Specifically, to account for the possible collinearity existing between FA values of consecutive segments along a given tract, the Beta-Binomial Sequential Goodness of Fit (BBSGoF; $k_{\min }=5$, $k_{\max }=8$, blocks $=5$ ) [42] was used, as proposed in the $\mathrm{R}$ package (https://cran.r-project.org/web/packages/sgof/sgof.pdf).

To better understand the nature of FA abnormalities in segment clusters showing a main effect of group upon FA in Level-2 analyses, mean AD and RD were extracted. Parallel ANCOVAs were used to examine a main effect of group upon these measures after accounting for age and gender.

Additional analyses. Mean FA was extracted in the segment clusters, i.e., clusters of contiguous segments within the left or right tract-profile of the cingulum bundle that showed a main effect of group or a main (simple or triple) interaction in Level-1 analysis, and that survived multiple comparison correction in Level-2 analysis. In adults with OCD, we then examined relationships between symptom severity (HRSD, HAMA, Y-BOCS, OC-TCDQ-incompleteness, OC-TCDQ-harm avoidance, and POPS in adults with OCD) and mean FA in segment clusters showing a significant main effect of group in Level-2 analyses, using six parallel repeated measure ANCOVAs, one for each symptom dimension as the betweensubjects effect, and FA in the two segment clusters (LATERALITY: 2 levels, i.e., the two segment clusters in the left and right cingulum bundle) as the within-subjects effect. Significant findings were then further examined using Pearson and/or Spearman correlations, as appropriate. The effect of SSRI (ON/OFF) or the effect of MDD comorbidity (yes/no) on FA in these segment clusters were also explored, using a similar approach. To account for multiple comparisons of FA-symptom dimension severity/medication in analyses focusing just on adults with OCD (i.e., analyses using the HRSD, HAMA, Y-BOCS, OC-TCDQ-incompleteness and OC-TCDQharm avoidance, POPS, MDD comorbidity, and SSRIs) the significance threshold was set at $P=0.05 / 8=0.006$.

Control tracts. Level-1-2 analyses were repeated for the left and right corticospinal tracts. To account for the examination of two white matter tracts in main analyses, the significance level was set at $P=0.05 / 2=0.025$ in Level- 1 analysis.

Other tracts. To explore the role of other major white matter tracts in the pathophysiology of OCD, Level-1-2 analyses were repeated for the left (50 segments) and right (50 segments) portion of the forceps minor and forceps major of the corpus callosum, the left and right anterior thalamic radiation, the left and right inferior longitudinal fasciculus, the left and right parietal and temporal portion of the superior longitudinal fasciculus, and the left and right uncinate fasciculus.

Scanner, age, and sex were covariates in all analyses.

Sensitivity analysis. Level-1-2 analyses were repeated after exclusion of data collected on the Trio.

\section{RESULTS}

Demographic and clinical characteristics

There were no significant group differences in age, sex ratio, SES, and predicted IQ. As expected, adults with OCD had significantly greater anxiety (HAMA), depressive (HRSD17) and OCD (Y-BOCS, OC-TCDQ-incompleteness, OC-TCDQ-harm avoidance, and POPS) 
symptom severity than CONT. In addition, 16 out of 44 adults with OCD were taking SSRIs (Table 1).

\section{Neuroimaging}

Level-1 analyses. After accounting for effects of scanner, age and sex, a repeated measure ANCOVA revealed a significant main effect of GROUP (between-subjects effect: $\mathrm{F}_{[1,87]}=5.3 ; P=0.024$ ) on FA on the left and right cingulum bundle. This analysis also revealed a trend effect of LATERALITY (within-subjects effect: $F_{[1,87]}=3.9$; $P=0.050$; which did not survive correction for multiple comparisons, $P=0.050 / 2=0.025$ to account for examination of the corticospinal tract, as a control tract; see above), and an effect of SEGMENT (within-subjects effect: $\mathrm{F}_{[1,87]}=5.4 ; P<0.001$ ) (Table 2).

\section{Level-2 analyses}

Main effect of group: After accounting for effects of scanner, age and sex, adults with OCD showed lower FA than CONT in the middle sections of the left and right cingulum bundle (OCD versus CONT in the left cingulum bundle: $k=29$ consecutive segments; OCD versus CONT in the right cingulum bundle: $k=17$ consecutive segments; $P<0.05$ ). In the left cingulum bundle, 22 out of these 29 consecutive segments survived correction for multiple comparisons $(P<0.030)$. In the right cingulum bundle, 15 out of these 17 consecutive segments survived correction for multiple comparisons $(P<0.028$; Fig. 1).

Further analyses revealed that adults with OCD showed higher RD than CONT in segment clusters of the left and right cingulum bundle; however, these findings were not significant $(P>0.05)$. There was no between-group difference in AD $(P>0.05)$.

\section{Additional analyses}

In adults with OCD: After accounting for effects of scanner, age, and sex, using six parallel repeated measure ANCOVAs (one for each symptom dimension), we did not find any relationship between mean FA in the segment clusters showing betweengroup differences in FA in the left or right cingulum bundle and symptom severity as measured by the HDRS-D, HAMA-A, Y-BOCS OC-TCDQ-incompleteness and OC-TCDQ-harm avoidance, and POPS in adults with OCD. We also did not find any effect of MDD comorbidity or SSRI on mean FA in these segment clusters in the left and right cingulum bundle (Supplemental Table 2).

Control tracts. After accounting for the effect of age and sex, a repeated measure ANCOVA found no effect of GROUP $\left(\mathrm{F}_{[1,91]}=2.7\right.$; $P=0.107)$, no effect of LATERALITY $\left(\mathrm{F}_{[1,91]}=0.5 ; P=0.938\right)$, and no LATERALITY by SEGMENT by GROUP interaction $\left(\mathrm{F}_{[99,5488]}=0.5\right.$; $P=0.695)$. Findings in the corticospinal tracts are reported in detail in Supplemental Table 3A.

Other tracts. In other major white matter tracts, there was a LATERALITY by SEGMENT by GROUP interaction $\left(\mathrm{F}_{[98,7938]}=1.8\right.$; $P=<0.001$ ) in the inferior longitudinal fasciculus. Further analyses revealed that adults with OCD showed lower FA that healthy adults in a middle portion ( 9 segments) of the left inferior longitudinal fasciculus. Mean FA in this segment cluster did not correlate with any symptom dimension, however (all $P>0.05$ ). These findings and findings on other major white matter tracts are reported in detail in Supplemental Table 3B.

Sensitivity analysis. Findings are reported in the Supplemental Table 4.

\section{DISCUSSION}

The goal of the present study was to use, for the first time, a tract-profile approach to examine the extent to which diffusion imaging abnormalities previously reported in the cingulum bundle of adults with OCD are focal or extended across the entire cingulum bundle. In support of previous studies [17, 30, 43-47], findings from this study showed lower collinearity of fibers in both the left and right cingulum bundle of adults with $O C D$ versus healthy adults. Using a tract-profile approach for the first time in OCD, our findings further demonstrate that lower FA in the cingulum bundle is focal, middle section of this tract. Further analyses suggested that in adults with OCD there might be a greater complexity of the fiber architecture and/or abnormal integrity of the fibers (greater RD) in comparison to healthy adults; however, this finding did not reach significance. It is probable that the focal abnormalities in this tract reflect abnormalities in the collinearity/integrity of specific fibers that connect the ACC with other prefrontal cortical regions. Indeed, fibers in these portions of the cingulum bundle have been shown to project not only to other portions of the ACC, including subgenual and dorsal ACC, but also to prefrontal cortex, including the orbitofrontal cortex, and subcortical regions, including the anterior ventral nucleus and lateral dorsal nucleus of the thalamus [6]. All these regions implicated in neural mechanisms underlying $\operatorname{OCD}[6,7,12,14]$.

To our knowledge, only two previous diffusion imaging studies employed similar tractographic approaches in OCD $[46,48]$. Neither of these studies reported diffusion imaging abnormalities in the cingulum bundle. In fact, one of these studies did not find any FA abnormality in any of the major white matter tracts examined [46]. The second study reported lower FA only in the forceps minor and in the right uncinate fasciculus [48]. Given that the cingulum bundle is a large white matter tract of $\sim 8002 \mathrm{~mm}^{2}$ voxels, it is possible that conventional approaches examining mean diffusivity parameters across the entirety of each tract might have precluded identification of any focal abnormality in the cingulum. Power might also be a factor, given that these studies included fewer than 30 patients with OCD.

As expected, we did not find any significant effect of group on FA in the corticospinal tracts in this study. In contrast with previous studies, however, examination of other major white matter tracts also did not reveal any other white matter microstructural abnormalities in adults with OCD (versus healthy adults). While the tract-profiles allow examination of white matter microstructure in segments along the length-the major axis-of a given tract, it is important to note that very large white matter tracts/regions, such as the corpus callosum or internal capsule, are known to have both a rostro-caudal and/or medial-lateral organization of fibers. For example, more ventral portions of the anterior limb of the internal capsule project to ventromedial/ ventrolateral portions of the prefrontal cortical regions, while dorsal portions of the anterior limb of the internal capsule project to dorsomedial/dorsolateral portions of the prefrontal cortical regions [49] Thus, tract-profile approaches are not suitable for the study of the microstructure of white matter tracts in which rostrocaudal and/or medial-lateral segmentations are needed.

Certain limitations of the study should be considered. Additional analyses did not reveal any relationship between FA abnormalities and severity of OCD symptoms as measured by the Y-BOCS, or with severity of other symptoms in adults with OCD at the time of the scan. It is important to note, however, that this sample size allowed us to explore linear relationships. Larger studies would allow us to model polynomial functions, which, in turn, might help identify more complex relationships between FA in the anterior segments of the cingulum bundle and severity of OCD symptoms. As mentioned above, our approach did not identify abnormalities in other major white matter tracts in which abnormalities in FA have previously been reported in individuals with OCD. Studies employing protocols for segmenting major white matter tracts are needed to further understand the contribution of white matter microstructural abnormalities in other tracts in OCD.

In summary, using a tract-profile approach for the first time in OCD, we demonstrate focal abnormalities in the middle portions 
a

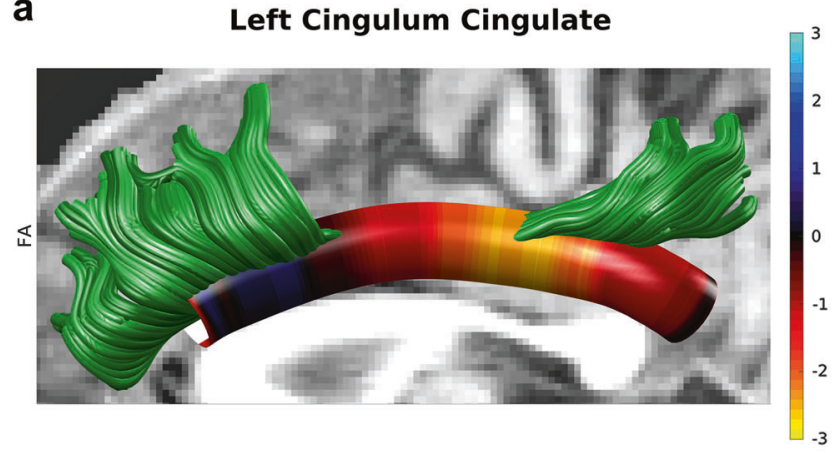

b

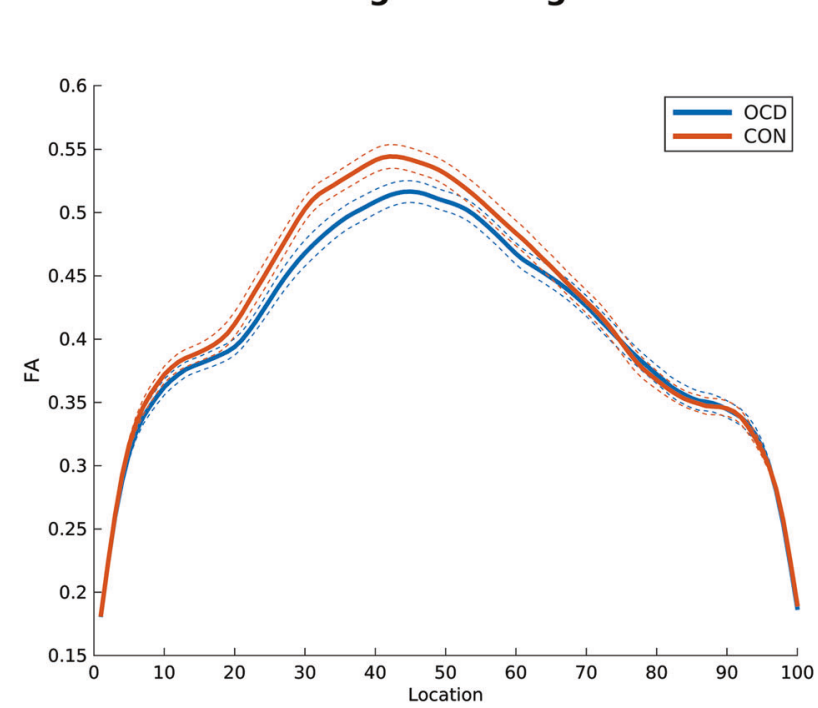

Left Cingulum Cingulate

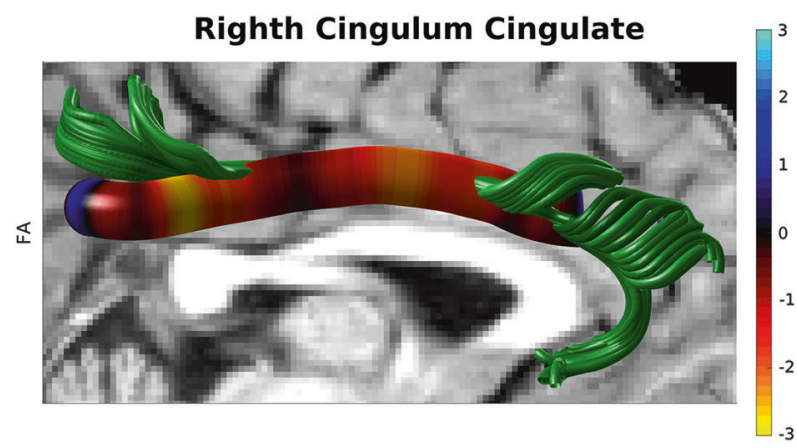

Righth Cingulum Cingulate

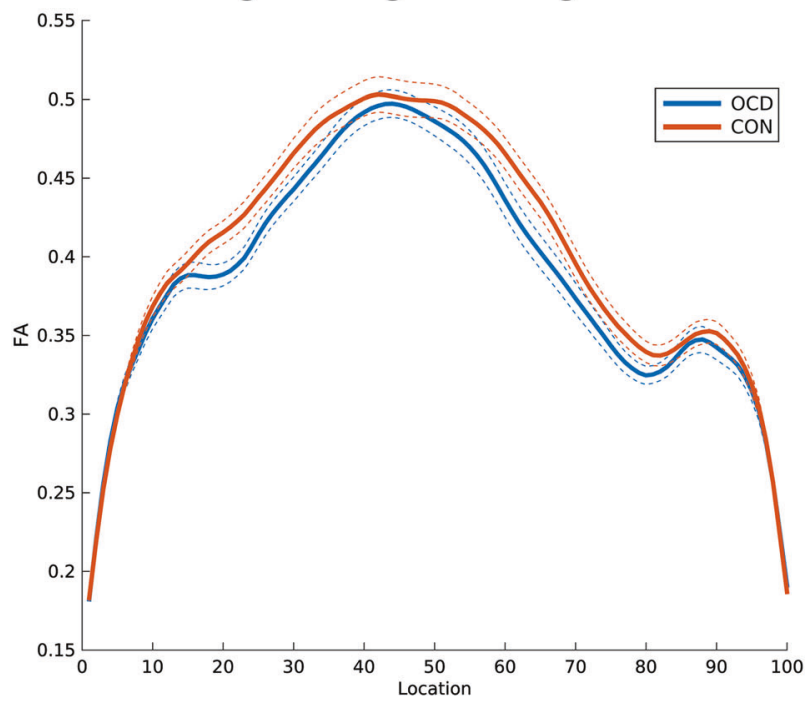

Fig. 1 a 3D visualization of the left and right cingulum bundle showing lower FA in OCD versus CONT. Tractographic analyses were performed in native space. Findings are displayed in one participant to represent between-group differences (red-yellow color bar depicts significance levels of $3>t>-3$ ). b Curve-trajectory plots represent the mean FA along the left and right cingulum bundle in OCD versus CONT. One hundred segments were used to define the tract-profile of the left and right cingulum in each tract separately. Confidence interval of $95 \%$ is represented with dotted lines. OCD obsessive-compulsive disorder, CONT healthy control individuals

of the left and right cingulum bundles in adults with OCD. The middle portion of the cingulum bundle carries fibers specifically between the ACC and other prefrontal and subcortical regions that have been proposed to have a key role in the pathophysiology of OCD. New protocols are needed to allow segmentation of other white matter tracts, such as the corpus callosum and anterior thalamic radiation/internal capsule, to help elucidate more focal neural mechanisms of OCD, to ultimately guide the development of new interventions for this debilitating disorder.

\section{FUNDING AND DISCLOSURE}

The present study was supported by the National Institute of Mental Health grant P50 MH106435 (PI: Dr. Haber). This funding agency was not involved in the design or conduct of the study, the collection, management, analysis, or interpretation of the data, or the preparation, review, or approval of the manuscript. The authors declare no competing interests.

\section{ACKNOWLEDGEMENTS}

We acknowledge Drs. Buckner and Quirk for their scientific contribution to this work. We also acknowledge our participants for their participation into this study.

\section{ADDITIONAL INFORMATION}

Supplementary Information accompanies this paper at (https://doi.org/10.1038/ s41386-019-0353-4)

Publisher's note: Springer Nature remains neutral with regard to jurisdictional claims in published maps and institutional affiliations.

\section{REFERENCES}

1. Ruscio AM, Stein DJ, Chiu WT, Kessler RC. The epidemiology of obsessivecompulsive disorder in the national comorbidity survey replication. Mol Psychiatry. 2010;15:53-63.

2. Nestadt G, Di CZ, Riddle MA, Grados MA, Greenberg BD, Fyer AJ, et al. Obsessive-compulsive disorder: subclassification based on co-morbidity. Psychol Med. 2009;39:1491-501.

3. Simpson HB, Foa EB, Liebowitz MR, Huppert JD, Cahill S, Maher MJ, et al. Cognitive-behavioral therapy vs risperidone for augmenting serotonin reuptake inhibitors in obsessive-compulsive disorder: a randomized clinical trial. Jama Psychiatry. 2013;70:1190-8.

4. Lopes AC, de Mathis ME, Canteras MM, Salvajoli JV, Del Porto JA, Miguel EC. Update on neurosurgical treatment for obsessive compulsive disorder. Rev Bras Psiquiatr. 2004;26:62-6.

5. Greenberg BD, Rauch SL, Haber SN. Invasive circuitry-based neurotherapeutics: stereotactic ablation and deep brain stimulation for OCD. Neuropsychopharmacology. 2010;35:317-36. 
6. Heilbronner SR, Haber SN. Frontal cortical and subcortical projections provide a basis for segmenting the cingulum bundle: implications for neuroimaging and psychiatric disorders. J Neurosci. 2014;34:10041-54.

7. Haber SN, Heilbronner SR. Translational research in OCD: circuitry and mechanisms. Neuropsychopharmacology. 2013;38:252-3.

8. Jung $\mathrm{HH}$, Kim $\mathrm{CH}$, Chang JH, Park YG, Chung SS, Chang JW. Bilateral anterior cingulotomy for refractory obsessive-compulsive disorder: Long-term follow-up results. Stereotact Funct Neurosurg. 2006;84:184-9.

9. Feldman RP, Alterman RL, Goodrich JT. Contemporary psychosurgery and a look to the future. J Neurosurg. 2001;95:944-56.

10. Cohen RA, Paul R, Zawacki TM, Moser DJ, Sweet L, Wilkinson H. Emotional and personality changes following cingulotomy. Emotion. 2001;1:38-50.

11. Tow PM, Whitty CW. Personality changes after operations on the cingulate gyrus in man. J Neurol Neurosurg Psychiatry. 1953;16:186-93.

12. Britton JC, Rauch SL, Rosso IM, Killgore WD, Price LM, Ragan J, et al. Cognitive inflexibility and frontal-cortical activation in pediatric obsessive-compulsive disorder. J Am Acad Child Adolesc Psychiatry. 2010;49:944-53.

13. Haber SN, Behrens TE. The neural network underlying incentive-based learning: implications for interpreting circuit disruptions in psychiatric disorders. Neuron. 2014;83:1019-39.

14. Aouizerate B, Rotge JY, Bioulac B, Tignol J. Present contribution of neurosciences to a new clinical reading of obsessive-compulsive disorder. L'Encephale. 2007;33:203-10.

15. Wu Y, Sun D, Wang Y, Wang Y, Ou S. Segmentation of the cingulum bundle in the human brain: a new perspective based on dsi tractography and fiber dissection study. Front Neuroanat. 2016;10:84

16. Bubb EJ, Metzler-Baddeley C, Aggleton JP. The cingulum bundle: anatomy, function, and dysfunction. Neurosci Biobehav Rev. 2018;92:104-27.

17. Koch K, Reeß TJ, Rus OG, Zimmer C, Zaudig M. Diffusion tensor imaging (DTI) studies in patients with obsessive-compulsive disorder (OCD): a review. J Psychiatr Res. 2014;54:26-35.

18. Radua J, Grau M, van den Heuvel OA, Thiebaut de Schotten M, Stein DJ, CanalesRodríguez EJ, et al. Multimodal voxel-based meta-analysis of white matter abnormalities in obsessive-compulsive disorder. Neuropsychopharmacology. 2014;39:1547-57.

19. Gruner P, Vo A, Ikuta T, Mahon K, Peters BD, Malhotra AK, et al. White matter abnormalities in pediatric obsessive-compulsive disorder. Neuropsychopharmacology. 2012;37:2730.

20. Jayarajan RN, Venkatasubramanian G, Viswanath B, Reddy YCJ, Srinath S, Vasudev MK, et al. White matter abnormalities in children and adolescents with obsessivecompulsive disorder: a diffusion tensor imaging study. Depress Anxiety. 2012;29:780-8.

21. Cannistraro PA, Makris N, Howard JD, Wedig MM, Hodge SM, Wilhelm S, et al. A diffusion tensor imaging study of white matter in obsessive-compulsive disorder. Depress Anxiety. 2007;24:440-6.

22. Sibilia F, Kehoe EG, Farrell D, Kerskens C, O'Neill D, McNulty JP, et al. Aging-related microstructural alterations along the length of the cingulum bundle. Brain Connect. 2017;7:366-72.

23. Jang $\mathrm{SH}$, Kwon $\mathrm{YH}$, Lee MY, Kim JR, Seo JP. Aging of the cingulum in the human brain: Preliminary study of a diffusion tensor imaging study. Neurosci Lett. 2016;610:213-7.

24. Mårtensson J, Lätt J, Åhs F, Fredrikson $M$, Söderlund H, Schiöth HB, et al. Diffusion tensor imaging and tractography of the white matter in normal aging: the rate-ofchange differs between segments within tracts. Magn Reson Imaging. 2018;45:113-9.

25. Lochner C, Fouche JP, du Plessis S, Spottiswoode B, Seedat S, Fineberg N, et al. Evidence for fractional anisotropy and mean diffusivity white matter abnormalities in the internal capsule and cingulum in patients with obsessive-compulsive disorder. J Psychiatry Neurosci. 2012;37:193-9.

26. Fontenelle LF, Bramati IE, Moll J, Mendlowicz MV, de Oliveira-Souza R, Tovar-Moll F. White matter changes in OCD revealed by diffusion tensor imaging. CNS Spectr. 2011;16:101-9.

27. Silk T, Chen J, Seal M, Vance A. White matter abnormalities in pediatric obsessivecompulsive disorder. Psychiatry Res. 2013;213:154-60.

28. Nakamae T, Narumoto J, Sakai Y, Nishida S, Yamada K, Nishimura T, et al. Diffusion tensor imaging and tract-based spatial statistics in obsessive-compulsive disorder. J Psychiatr Res. 2011;45:687-90.

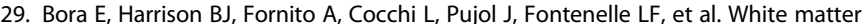
microstructure in patients with obsessive-compulsive disorder. J Psychiatry Neurosci. 2011;36:42-6.

30. Garibotto V, Scifo P, Gorini A, Alonso CR, Brambati S, Bellodi L, et al. Disorganization of anatomical connectivity in obsessive compulsive disorder: a multi-parameter diffusion tensor imaging study in a subpopulation of patients. Neurobiol Dis. 2010;37:468-76.

31. Wang R, Fan Q, Zhang Z, Chen Y, Zhu Y, Li Y. Anterior thalamic radiation structural and metabolic changes in obsessive-compulsive disorder: a combined DTIMRS study. Psychiatry Res Neuroimaging. 2018;277:39-44.

32. Yoo SY, Jang JH, Shin Y-W, Kim DJ, Park H-J, Moon W-J, et al. White matter abnormalities in drug-naïve patients with obsessive-compulsive disorder: a Diffusion Tensor Study before and after citalopram treatment. Acta Psychiatr Scand. 2007;116:211-9.

33. Yakovlev PI, Locke SS, Koskoff DY, Patton RA. Limbic nuclei of thalamus and connections of limbic cortex. Arch Neurol. 1960;3:620-41.

34. Nelson HE, Willison JR (1991). The revised National Adult Reading Test-Test Manual. Windsor, UK: Nfer-Nelson WUK.

35. Goodman WK, Price LH, Rasmussen SA, Mazure C, Delgado P, Heninger GR, et al. The yale-brown obsessive compulsive scale: li. validity. Arch Gen Psychiatry. 1989;46:1012-6.

36. An SK, Mataix-Cols D, Lawrence NS, Wooderson S, Giampietro V, Speckens A, et al. To discard or not to discard: the neural basis of hoarding symptoms in obsessivecompulsive disorder. Mol Psychiatry. 2009;14:318-31.

37. Andersson JL, Skare S, Ashburner J. How to correct susceptibility distortions in spin-echo echo-planar images: application to diffusion tensor imaging. Neuroimage. 2003;20:870-88.

38. Smith SM, Jenkinson M, Woolrich MW, Beckmann CF, Behrens TEJ, Johansen-Berg $\mathrm{H}$, et al. Advances in functional and structural MR image analysis and implementation as FSL. Neuroimage. 2004;23:S208-19.

39. Yendiki A, Koldewyn K, Kakunoori S, Kanwisher N, Fischl B. Spurious group differences due to head motion in a diffusion MRI study. Neuroimage. 2014;88:79-90.

40. Yeatman JD, Dougherty RF, Myall NJ, Wandell BA, Feldman HM. Tract profiles of white matter properties: automating fiber-tract quantification. PLoS ONE. 2012;7: e49790.

41. Jones DK, Leemans A. Diffusion tensor imaging. Methods Mol Biol. 2011;711:127-44.

42. Castro-Conde I, de Uña-Álvarez J. Adjusted p-values for SGoF multiple test procedure. Biom J. 2015;57:108-22.

43. Admon R, Bleich-Cohen M, Weizmant R, Poyurovsky M, Faragian S, Hendler T. Functional and structural neural indices of risk aversion in obsessive-compulsive disorder (OCD). Psychiatry Res. 2012;203:207-13.

44. Ha TH, Kang D-H, Park JS, Jang JH, Jung WH, Choi J-S, et al. White matter alterations in male patients with obsessive-compulsive disorder. Neuroreport. 2009;20:735-9.

45. Szeszko PR, Ardekani BA, Ashtari M, et al. White matter abnormalities in obsessive-compulsive disorder: a diffusion tensor imaging study. Arch Gen Psychiatry. 2005;62:782-90.

46. Watanabe A, Nakamae T, Sakai Y, Nishida S, Abe Y, Yamada K, et al. The detection of white matter alterations in obsessive-compulsive disorder revealed by TRActs Constrained by UnderLying Anatomy (TRACULA). Neuropsychiatr Dis Treat. 2018;14:1635-43.

47. Benedetti F, Giacosa C, Radaelli D, Poletti S, Pozzi E, Dallaspezia S, et al. Widespread changes of white matter microstructure in obsessive-compulsive disorder: effect of drug status. Eur Neuropsychopharmacol. 2013;23:581-93.

48. He X, Steinberg E, Stefan M, Fontaine M, Simpson HB, Marsh R. Altered frontal interhemispheric and fronto-limbic structural connectivity in unmedicated adults with obsessive-compulsive disorder. Hum Brain Mapp. 2018:39:803-10.

49. Safadi Z, Grisot G, Jbabdi S, Behrens T, Heilbronner SR, McLaughlin NCR, et al. Functional segmentation of the anterior limb of the internal capsule: linking white matter abnormalities to specific connections. J Neurosci. 2018;38:2106-17.

Open Access This article is licensed under a Creative Commons Attribution 4.0 International License, which permits use, sharing, adaptation, distribution and reproduction in any medium or format, as long as you give appropriate credit to the original author(s) and the source, provide a link to the Creative Commons license, and indicate if changes were made. The images or other third party material in this article are included in the article's Creative Commons license, unless indicated otherwise in a credit line to the material. If material is not included in the article's Creative Commons license and your intended use is not permitted by statutory regulation or exceeds the permitted use, you will need to obtain permission directly from the copyright holder. To view a copy of this license, visit http://creativecommons. org/licenses/by/4.0/.

(c) The Author(s) 2019 